\title{
Mapping of equipotential surfaces using the free Quantum Geographic Information System software
}

\author{
H. Finatto ${ }^{1}$, G. H. M. Voigt ${ }^{1}$, B. C. Carvalho ${ }^{1}$, L. B. Reyna Zegarra ${ }^{3}$, L. E. G. Armas*2@ \\ ${ }^{1}$ Universidade Federal do Pampa, Alegrete, RS, Brasil \\ ${ }^{2}$ Universidade Federal do Pampa, Grupo de Óptica, Micro e Nanofabricação de Dispositivos, Alegrete, RS, Brasil \\ ${ }^{3}$ Universidad Nacional del Santa, Departamento de Matemática, Chimbote, Perú
}

Received on March 05, 2019; Revised on April 07, 2019; Accepted on May 03, 2019.

\begin{abstract}
In this work, we report the mapping of electrical equipotential lines (1D) and equipotential surfaces (3D) using the free Quantum Geographic information system (QGIS) software. For this purpose, experiments taking into account, four different electrical configurations were performed on physics classes of undergraduate students, using two conductors of opposite electrical charges for each experiment. For the first experiment two copper parallel linear conductors; for the second, a copper parallel linear conductor with a small circular ring acting as a point charge; for the third, two concentric circular ring and for the fourth one a semicircular ring with a small circular ring acting as point charge. The experimental data were treated and interpolated in the, open source, QGIS software, used in geoprocessing, to map the electrical equipotential planes and surfaces.

Keywords: Mapping, Equipotential lines, Equipotential surfaces, Electric potential, Geospatial Software - QGIS.
\end{abstract}

\section{Introduction}

The experimental practices of physics are instrumental options for demonstration and identification of concepts related to physics classes. The experiments performed on laboratory can be a tool of theoretical exemplification, illustrating the physical facts of study. Nowadays, the teaching of physics use a wide range of strategies and free softwares for discussion and approach of concepts related to different physics phenomenon. For instance, Marin et al. [1] have used the Tracker freeware software for the study of experiments in electricity and magnetism using analog instruments for electrical signal measurements. The Tracker is a piece of freeware software, designed to use video recorded images of the motion of objects as input data, and has been mostly applied in physics education to analyze and simulate physical phenomenon in mechanics. Mandanici [2] has used the GNU Octave Scientific Programming Language for the study of the transient phenomena in the series RLC circuit, the dispersion of light through a prisms, drawing of the damped mechanical oscillator and others.

Up to now there are no studies about mapping of electrical equipotential lines and surfaces using the Quantum Geographic information system (QGIS) software. The few studies are based on computational methods and well known open source softwares. For instance, Young [3] has used computational methods based on relaxation method for graphing nonphysical equipotential lines obtained with the electric-field mapping experiment. Richards [4]

*Correspondence email address: legarmas@gmail.com. has plotted equipotential and electric field lines using the method of semi silvered mirror for plotting orthogonal curves. This method consists of drawing a normal to a curve using a front silvered mirror. On the other hand, Marshand [5], of the Northern Illinois University Physics Department, has shown to the students class the mapping of equipotential lines and surfaces using the Excel spreadsheet.

On the other hand, QGIS (previously known as Quantum GIS) is a free and open-source cross-platform desktop geographic information system application that supports viewing, and analysis of geospatial data $[6,7,8,9]$, and up to now it has not been used on the mapping of electrical equipotential surfaces. Therefore, in this work, it is proposed, an experimental approach for mapping equipotential surfaces with different charge distributions (two parallel linear conductors; a parallel linear conductor with a small ring circular acting as a point charge; two concentric circular conductors and a semicircular ring with a small circular ring acting as point charge) at the presence of an electrolytic solution of $\mathrm{NaCl}$. The data were processed in the QGIS software [6], obtaining the interpolation of the experimental data and extraction of the equipotential surfaces, for later analysis of the electric fields distribution for each proposed geometry.

\section{The experiments}

The experiments were carried out during laboratory classroom lectures for engineering at the Federal University of Pampa- UNIPAMPA-Campus Alegrete, RS, Brasil. The 
students were enrolled in a first semester introductory physics III course. This course covers electricity and magnetism and it is taken after the first introductory physics III course, which covers electrical fields and electrical potential. The experiments were taught to 40 students divided into five groups. Each group had $2 \mathrm{~h}$ of laboratory classroom work per week. The four experiments presented in this work are part of one experiment performed during the semester. The students presented a written report weekly.

Before the laboratory work the students were trained in the installation and use of Geospatial software in a $1.5 \mathrm{~h}$ lecture. The use of Geospatial software helped to overcome some limitations during mapping of the equipotential surfaces.

For the experimental setup was used a well-known practice for determination of equipotential surfaces [10-13]. The materials used were copper hard wire, electrolytic solution (water - $600 \mathrm{ml}$ and salt - $80 \mathrm{~g}$ ), multimeter, direct current source, graph paper and acrylic plate (electrolytic cell). The electrode configurations chosen for this experiment are shown in Figs. 1(a)-(d). In all configurations the reference point of the multimeter was connected to the negative source output (marked with letter $\mathrm{N}$ in all configurations).

Figure 1(a) shows an electrolytic configuration of a parallel plate capacitor arranged on a system of coordinate
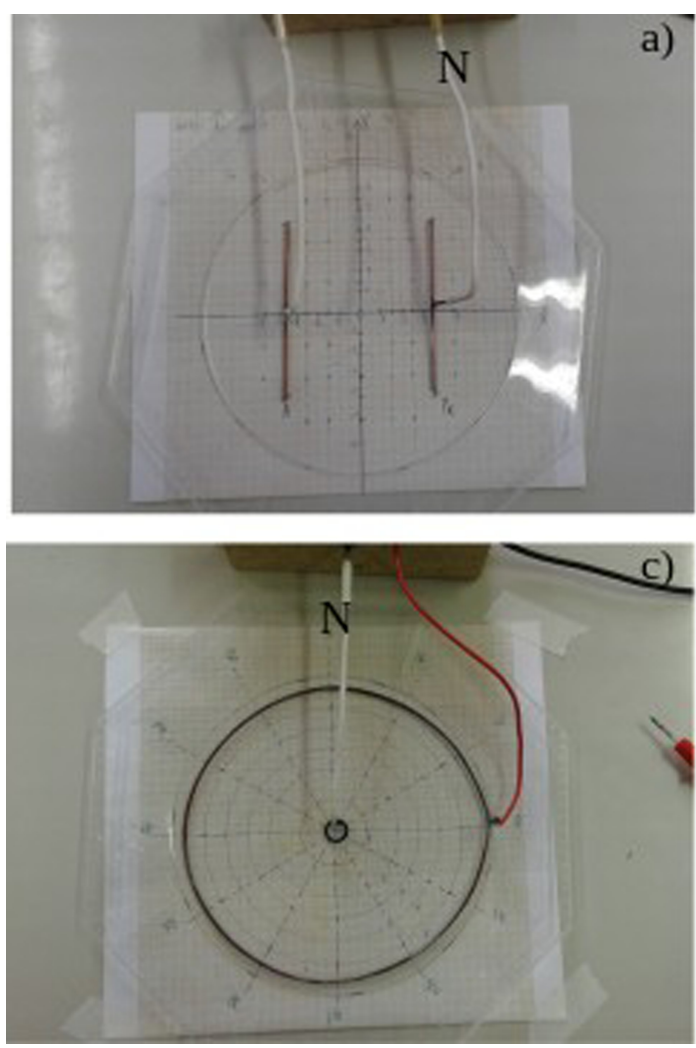

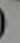
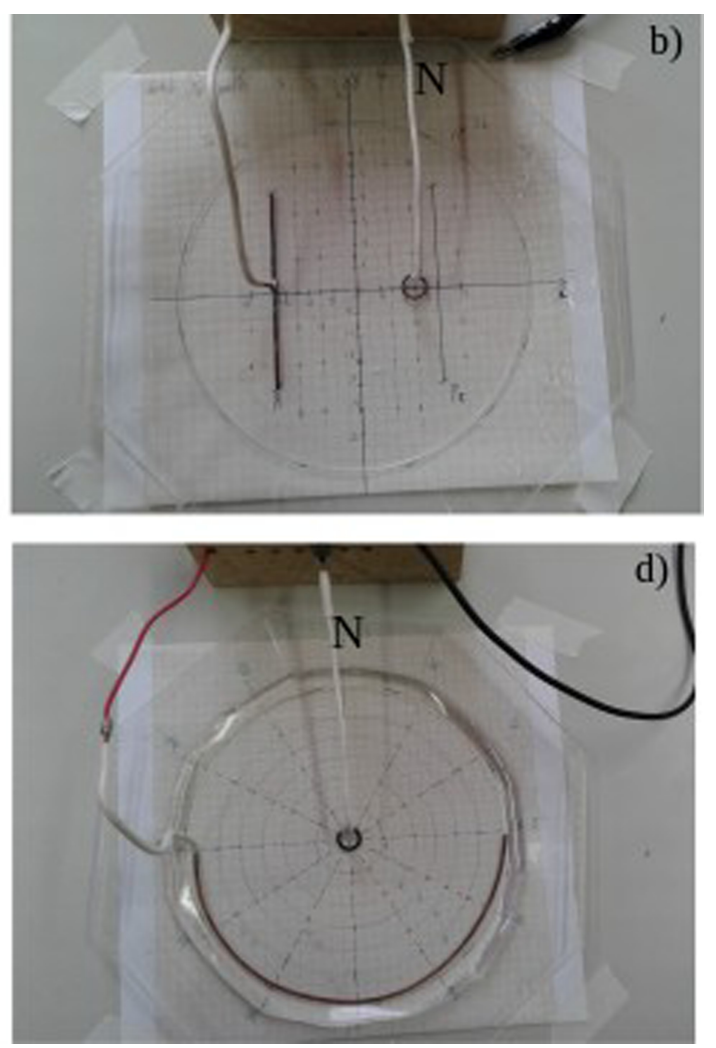

Figure 1: Experimental set up of the electrode configurations: (a) parallel linear conductors (parallel plate capacitor); (b) parallel conductor and a small circular ring acting as a negative point charge; (c) two concentric circular rings and (d) a semicircular ring with a small circular ring acting as point charge. Letter $\mathrm{N}$, in all configurations, represents the negative charge. axis; Fig. 1(b) shows the experimental arrangement of a positively charged plate and a point charge, represented by a small circular ring, referenced in space; Fig. 1(c) provides an arrangement of two circular electrodes, where the central ring represents a point charge, connected to the negative output of the voltage source, while the external ring is positively charged, both referenced in the experimental space. Finally, Fig. 1(d) shows a semicircular ring, positively charged and a point charge located at the center of this semicircle, which is connected to the negative output of the voltage source. For all measurements settings, the voltage source was adjusted to $16 \mathrm{~V}$.

\section{Data mapping}

In order to map the experimental data, in all experimental configurations, the reference terminal of the multimeter was connected to the negative output of the voltage source, whereas the other terminal was used as a mobile probe to measure the electrical potential at each point of interest. In order to obtain the experimental data two reference systems were used in this experiment.

- Rectangular system: the points for mapping were demarcated according to reported on the literature $[3,14]$, considering equidistant points of $1 \mathrm{~cm}$, for the arrangement of parallel plates (Fig. 1(a); the

d) 
same points were mapped in the arrangement of plate and point charge, as shown on Fig. 1(b), but considered random because the expected geometry of the surfaces differs from the previous one. The voltage probe was moved in an area of $9 \mathrm{~cm} \times 9 \mathrm{~cm}$, collecting the potential difference at 45 different points between the electrodes.

- Polar system: used for the experimental demarcation of the equipotential surfaces of the array formed by the ring and point charge (Fig. 1(c); as well as for the semicircular configuration and a point charge (Fig. 1(d), the experimental points were considered random when mapped due to the lack of symmetry, but the results are in accordance with theory as it will be shown in the next section. In this case, the voltage probe was moved in an area of $12 \mathrm{~cm} \mathrm{x} 12 \mathrm{~cm}$, mapping 60 points, between the electrodes.
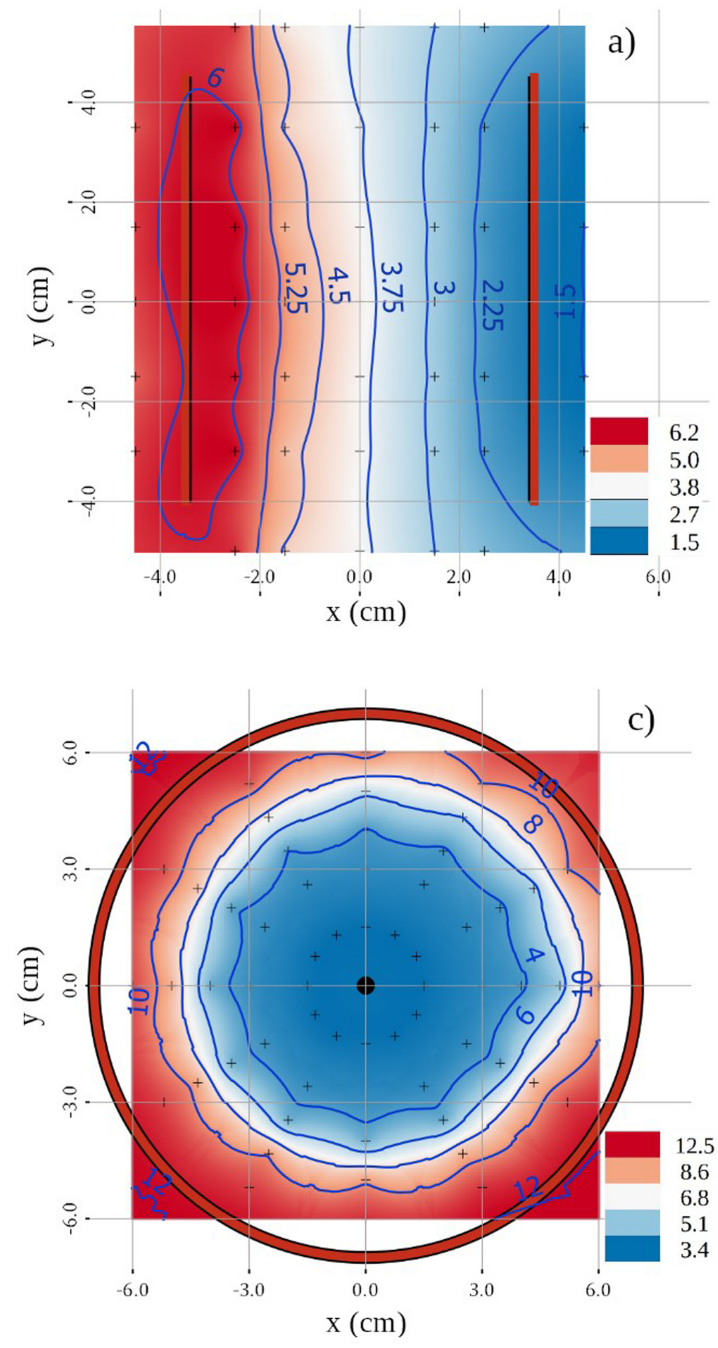

The data were stored with their spatial location in spreadsheets. The polar coordinate system was converted to rectangular coordinate because the software uses reference mesh of rectangular axes.

\section{Data processing with QGIS Software}

The QGIS software is a free and open source Geographic Information System. Data entry in QGIS was accomplished by adding a new layer delimited by text and stored in shapefile files. The data collected in the laboratory were interpolated individually, for each electrode configuration, and recorded in raster files. The interpolation method chosen was that of Kriging, which observes that near points in space have values closer than farther away points [15]. During the interpolation process, in the calculus, 30 points were taken into consideration in each pixel.
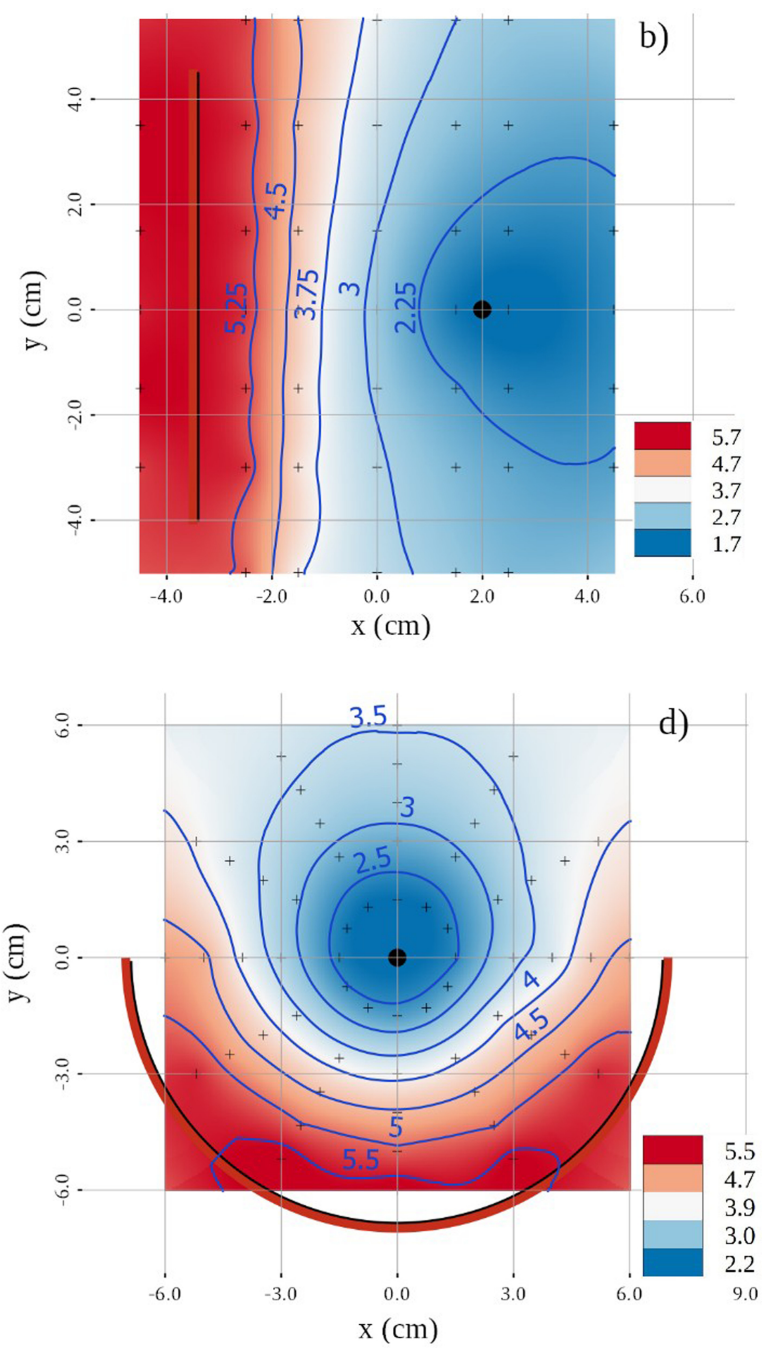

Figure 2: Color maps of the electric potential difference, for each configuration, obtained from the interpolated experimental data. Configurations of Fig. 2 ( $a, b, c$ and d) are in correspondence with configurations on Fig. 1 ( $a, b, c$ and d). Brown bars indicate the electrodes location, black dots represent the point charges, laboratory-mapped points are represented by crosses, blue colors indicate regions of low electrical potential and red color regions of high electrical potential. 
For the extraction of the equipotential surfaces, a QGIS routine was used to obtain contours of raster files, and were performed with an considered equidistance and appropriated for each configuration.

The data presentation in three dimensions was performed by means of a complement, which can be added to the QGIS used, called Qgis2threers. This complement generates third-dimension views using the tree.js JavaScript library [16].

\section{Results and Discussion}

Figure 2 shows the interpolated data, of the potential differences maps obtained from the laboratory-mapped data, which are marked with crosses and delimited by blue lines for each electrode configuration shown in Fig. 1 ( $\mathrm{a}, \mathrm{b}, \mathrm{c}$ and $\mathrm{d}$ ). For each electrode configuration the blue color corresponds to lower electrical potential $(\sim$ $1.5 \mathrm{~V}$ (Fig. 2(a), $1.7 \mathrm{~V}$ (Fig. 2(b), $3.4 \mathrm{~V}$ (Fig. 2(c) and $\sim 2.2 \mathrm{~V}$ (Fig. $2(\mathrm{~d}))$ and the red color corresponds to higher electrical potential $(\sim 6.2 \mathrm{~V}$ (Fig. $2(\mathrm{a}), \sim 5.7 \mathrm{~V}$ (Fig. 2(b), $12.5 \mathrm{~V}$ (Fig. 2(c) and $\sim 5.5 \mathrm{~V}$ (Fig. 2(d)), whereas white color corresponds to a intermediate value of electrical potential. These values are shown on the scale bar of each configuration on Fig. 2. The interpolation results for each experimental situation illustrate the actual situation found in the laboratory. The fancy color gradient helps in identifying the spreading of the electric potential associated with each situation. It is observed that the geometry of the electrodes interfere with the distribution of the extracted equipotential maps. For instance, for the configurations shown in Fig. 2(a, c), the distribution of the equipotential surfaces is more homogeneous than for the configurations of Fig. 2(b, d), causing that the electrical potential difference varies more rapidly in the region between the oppositely charged electrodes.

Figure 3 shows in detail the equipotential lines shown in Fig. 2, for each experimental situation, with the directions and orientations of the electric fields. As we can see, for all configurations the equipotential lines are always
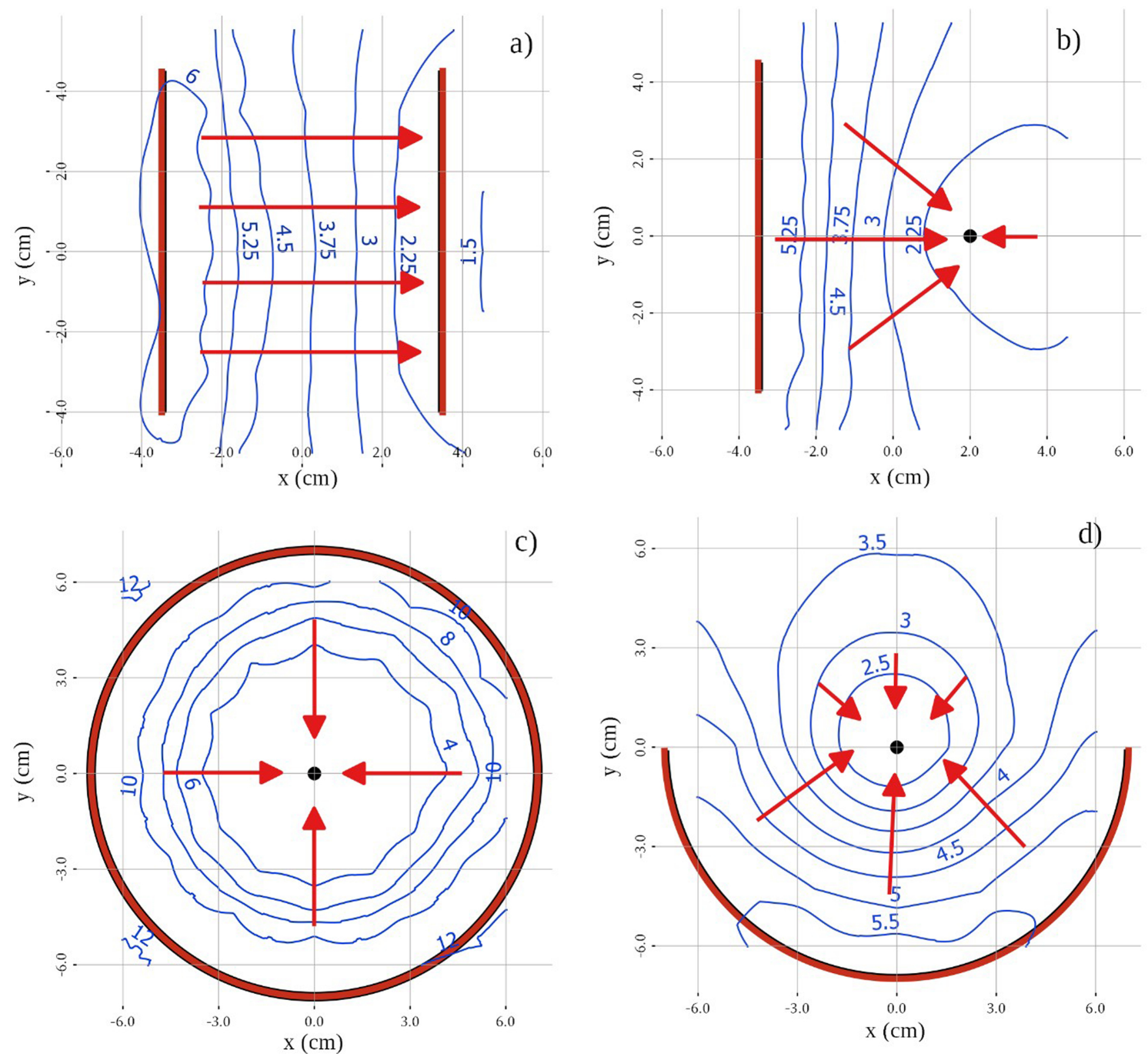

Figure 3: General orientation of the direction and intensity of the electric field, for each experimental configuration, indicated by red arrows, based on the distribution of equipotential lines, which are shown by blue lines. 
perpendicular to the electric field lines, and the geometry of the electric charge distribution influences directly on the geometry of the distribution of the equipotential lines, as reported on the literature [17-19]. For instance, if the charges are two parallel linear conductors (Fig. $3(\mathrm{a})$ ), the equipotential lines are parallel to these charges, similarly if the charges are two concentric circular rings the equipotential lines are also concentric rings to these charges, as shown in Fig. 3(c). On the other hand, if the charge distributions are not symmetric, as shown in Fig. 3(b), the electric field is more intense in the regions with higher density of equipotential lines and, in turn, weaker in regions with less equipotential lines. The physical phenomenon shown in Fig. 3(b) can also be seen in Fig. 3(d), where the greatest density of equipotential lines lies in the central region between the electrodes, creating a non-uniform electric field in the region between the electrodes. The potential difference between each potential lines for each configuration are given by: (a) 0.75 $\mathrm{V}$, (b) $0.75 \mathrm{~V}$, (c) $2 \mathrm{~V}$ and (d) $0.5 \mathrm{~V}$. Similar equipotential lines were reported by J. Elizalde and et al. [20] on parallel linear electrodes and a point charge with a circle electrode using a conductive gel for the plotting of equipotential lines. It is worth to emphasize that, the experimental conditions of this proposal, unfortunately, do not present an automatic and instantaneous form of the collection of the experimental data, being this manual from point to point. Thus, the post-processing of the data in the QGIS software illustrates the predicted theoretical conditions, but with some discontinuities, such as the non-smooth curves shown in Figs. 2 and 3. Even so, it is possible to see a trend smooth in each equipotential surface, on the various electrolytic configurations, as shown in Fig. 4.

In order to show the potential applications of the use of QGIS software in physical phenomena, besides being used as a geographic information system [21], Fig. 4 shows the mapping of the equipotential surfaces, obtained from the interpolated experimental data, of each contour surface (2D) shown in Fig. 2. The colors, potential values and values of $\mathrm{x}$, y coordinates shown on the $3 \mathrm{D}$ equipotential surfaces are in correspondence with the shown on the $2 \mathrm{D}$ color maps, shown in Fig. 2.
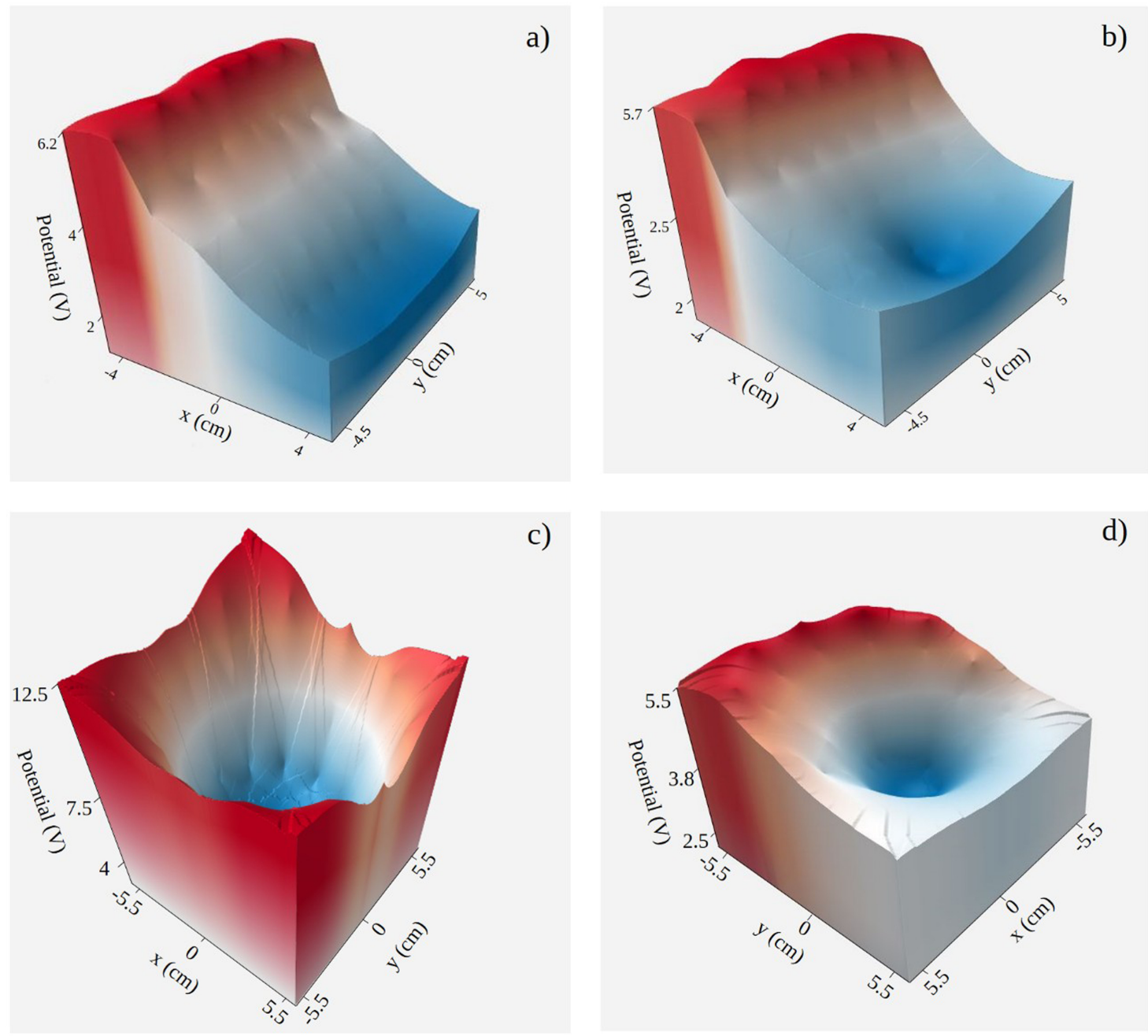

Figure 4: Equipotential surfaces (3D) corresponding to equipotential maps shown in Fig. 2. (a) parallel plates, (b) plate and point charge, (c) circular ring and point charge and (d) semicircular ring and point charge. The color scale follows the same orientation as the captions shown in Fig. 2. 
As Russell proposes an analogy with the gravitational hills to understand Einstein's law of gravitation [11], we can observe a very similar relationship between the gravitational hills, and the third dimension models shown on Fig. 4. Following the same reasoning used for relief shapes of a mountains landscape, we can relate the regions with different heights with the electric potential surfaces, observing the higher heights as the regions with greater potential difference, while depressions, as regions with smaller potential difference in relation to the reference point. Thus, blue regions of the equipotential surfaces, shown on Fig. 4(a, b, c and d), represent regions of low electrical potential (depressions), evidencing the need to perform a work to move any electrical charge from a region with lower electrical potential to a region with a higher electrical potential. This fact is similar to the work that must be performed by an extern agent to move an object from a depression to a certain height. A particularity on Fig. 4(b) is the lower electrical potential on the ring position than on its vicinity. On the other hand, the jagged appearance of the contours on the equipotential surfaces of a circular ring with a point charge (Fig. 4(c)), and the semicircular ring with a point charge (Fig. 4(d)) is due to the experimental data interpolation process. That is, the interpolated data were obtained only within the selected electrolytic configuration, leaving out interpolation from the edge to outside. This kind of interpolation is also reflected on the colors perpendicular to the $\mathrm{x}$ and $\mathrm{y}$ axis, which do not have any relation with potential difference, the colors related with potential difference are those perpendicular to the potential axis, beginning from the bottom (lower potential-blue color) to the top (higher potential-red color). These results are in accordance with the literature, for instance, Young [3] has reported the $3 \mathrm{D}$ potential surface of a $2 \mathrm{D}$ capacitor, considering two parallel thin electrodes each $6 \mathrm{~cm}$ long and separated by $2 \mathrm{~cm}$.

In the same way, the equipotential surface shown in Fig. 4(c), presents as a large well of electrical potential difference, with a low voltage central region surrounded by regions with higher voltages. It can be observed that to carry any point charge from a point located in the center to any one of the edges of the experimental configuration, the same amount of work is required, due to the symmetry of the presented configuration. On the other hand, the equipotential surface of the arc with point charge, shown in Fig. 4(d), also shows a well of potential energy. However, depending on the path to be considered, less energy is required to move a charge in the system. For instance, if the path considered goes to regions in red it would be necessary to have more energy than if the path considered is in the opposite direction.

\section{Conclusions}

In summary, we have reported that the range of applications of the Quantum Geographic information system (QGIS) software (so far mostly used in the field of civil engineering), can easily be extended to the study of electricity and magnetism experiments, related with both equipotential maps and surfaces. For this purpose, the mapping of electrical potential maps (2D) and surfaces (3D) of four electrical configurations were performed, showing that the distribution of the electrical potential depends of the experimental configuration between the electrodes. Therefore, the experiments carried out in this work, with the QGIS software as a tool, can be performed with easily found and low-cost equipment and materials and are simple to perform in schools and universities.

\section{Acknowledgments}

We are very grateful with the physics laboratory technicians of the Federal University of Pampa - UNIPAMPA, campus Alegrete, by the technical assistance during the performed of the experiments.

\section{References}

[1] P.A. Marin, M.E.C. Bacilio and S.R. Rosas, Eur. J. Phys. 39, 3 (2018).

[2] A. Mandanici, Eur. J. Phys. 39, 5 (2018).

[3] R.A. Young, Com. Phys 12, 432 (1998).

[4] A.D. Richards Phys. Educ. 9, 4 (1974).

[5] https://cpb-us-e1.wpmucdn.com/sites.pc.gsu.edu/ dist/c/891/files/2016/02/ElectrostaticField1 i5zqlv.pdf, accessed in november 2018.

[6] https://qgis.org/pt_BR/site/index.html.

[7] S.S.S. Sardeiro, Modelagem digital de terreno do município de Graccho Cardoso, nordeste de Sergipe. Masters Dissertation, Universidade Federal de Sergipe, Sergipe (2016).

[8] V.A.G. Gutiérres, R. Elect. Mat. 1, 46 (2016).

[9] D.P.A. Oliveira, E. Oliveira, E.M. Lima, J.B. Santos, M.R. Maia and R.S. Conceição, in Anais do IV Simpósio Geotecnologia e Geoinformação no Estado de Alagoas, Maceió, 2016 (Secretaria de Estado do Planejamento, Gestão e Patrimônio, Maceió, 2016).

[10] G. Beilner and F. Muchenski, Ens. Pesq 13, 75 (2015).

[11] V.R. Silva, A.M. Almeida, G.P.C. Campelo and A.C.L. Moreira, in Anais do III Congresso Nacional de Educação, Natal, 2016, (Realize, Campina Grande, 2016).

[12] D.S.D. Caetano, A. Cardoso, E. Lamounier Jr., in Anais do Workshop de Realidade Virtual e Aumentada, Bauru, 2008, (UNESP, Bauru, 2008).

[13] T.B. Batalhão, M.F. Cobo, E.R. Azevêdo, L.A.O. Nunes and L.G. Marcassa, Laboratório de Física III: livro de práticas (Instituto de Física de São Carlos, São Carlos, 2013), p. 47.

[14] J.A. Philips, S. Sanny, D. Derube and A. Hoemke, Phys. Teac. 55, 71 (2017).

[15] V. Olaya. Sistemas de Información Geográfica, available in https://github.com/volaya/libro-sig/releases/ download/v2.0/Libro_SIG.pdf

[16] QGIS Python Plugins Repository, available in https://plugins.qgis.org/plugins/Qgis2threejs/.

[17] R.A. Serway and J.W. Jewett Jr., Princípios de Física (Thomson Learning, São Paulo, 2006), v. 3, p. 722. 
[18] D. Halliday, in: Fundamentos de Física: eletromagnetismo (LTC, Rio de Janeiro, 2009), v. 3, p. 81.

[19] P.A. Tipler and G. Mosca, Física para Cientistas e Engenheiros (LTC, Rio de Janeiro, 2006), v. 2, p. 88.

[20] J.E. Torres, M.G. Cardel, E.J.V. Murguía, I.C. González and M.R. Nava, Phys. Educ. 50, 468 (2015).

[21] P. Longley, M. Goodchild, D. Maguire and D. Rhind, Geographic Information Systems and Science (Wiley, New Jersey, 2011). 